\title{
A importância das atividades educativas na conscientização do câncer de próstata:
}

\section{novembro azul}

The importance of educational activities in prostate cancer awareness: blue November

La importancia de las actividades educativas en la concienciación sobre el cáncer de próstata: noviembre azul

Ana Karina Rodrigues Coelho ORCID: https://orcid.org/0000-0001-8504-689X Centro Universitário Fibra, Brasil E-mail: anna.karinna1999@gmail.com João de Souza Mendonça ORCID: https://orcid.org/0000-0002-2204-5010

Universidade da Amazônia, Brasil E-mail:joaosm.farmaco@gmail.com Amanda Almeida Vieira ORCID: https://orcid.org/0000-0003-2211-0115

Centro Universitário do Estado Pará, Brasil E-mail: amandaav2000@gmail.com Marcello Vieira dos Santos ORCID: https://orcid.org/0000-0002-3356-1619 Centro Universitário do Estado do Pará, Brasil E-mail:marcellosantos277@gmail.com

Leidiane de Jesus da Costa Santos ORCID: https://orcid.org/0000-0002-9208-2319 Escola Superior da Amazônia, Brasil E-mail:leidianesantos2023@gmail.com

Tâmia Rayara Carvalho Araújo da Silva ORCID: https://orcid.org/0000-0003-4713-7314

Universidade da Amazônia, Brasil E-mail:ta.mia10@hotmail.com

Tamires Costa Franco ORCID: https://orcid.org/0000-0002-8978-4176

Universidade da Amazônia, Brasil

E-mail:tamiresfranco98@gmail.com

Maria Eduarda Monteiro Pereira ORCID: https://orcid.org/0000-0002-3679-9120

Centro Universitário do Estado do Pará, Brasil

E-mail: mariamontpereira@outlook.com

Débora Hosana Biasi

ORCID: https://orcid.org/0000-0003-0079-3963

Centro Universitário do Estado do Pará, Brasil E-mail: deborabiasi7@gmail.com Izadora Avelar Neto

ORCID: https://orcid.org/0000-0002-3481-2088 Universidade do Estado do Pará, Brasil E-mail: izadoraavelar@gmail.com

João Victor Álvares Guzzo

ORCID: https://orcid.org/0000-0002-1924-5409

Centro Universitário do Estado do Pará, Brasil E-mail: joao.guzzo11@gmail.com

Daniely Maués Beliqui ORCID: https://orcid.org/0000-0002-4191-2204 Centro Universitário do Estado do Pará, Brasil

E-mail: danielymauesbeliqui@gmail.com

Cassiane da Silva Portela Pinto

ORCID: https://orcid.org/0000-0002-5732-8555 Universidade do Estado do Pará, Brasil E-mail: cassiane_portela@yahoo.com

Márcia Mayanne Almeida Bezerra ORCID: https://orcid.org/0000-0002-5017-2914 Faculdade de Medicina Estácio de Castanhal, Brasil E-mail: marciamayanne1@gmail.com 


\title{
Resumo
}

O câncer de próstata é o tipo mais comum entre os homens. A próstata é uma glândula que só o homem possui e que se localiza na parte inferior do abdômen. Trata-se de um estudo de caráter descritivo, do tipo relato de experiência, realizado em uma unidade básica de saúde do Estado do Pará. Sabe-se que a uma baixa adesão na realização dos exames prescritos para detecção precoce do câncer de próstata, além da resistência dos homens em dar continuidade nas consultas periódicas, sendo assim, foi realizado uma atividade com intuito de desenvolver o processo saúde doença, para atender ao público alvo específico, homens em idade de rastreio do câncer de próstata. Teve como intenção adquirir um maior número de adesão dos pacientes da unidade de saúde do sexo masculino ao rastreamento. Sendo assim, a assistência multiprofissional é importante para que se possa buscar todos os eixos de ensino para ser repassado à comunidade, de modo que as ações educativas em conjunto esclarecem sobre as formas de prevenção e proporcionam um diagnóstico precoce.

Palavras-chave: Câncer; Educação em saúde; Multiprofissional.

\begin{abstract}
Prostate cancer is the most common type among men. The prostate is a gland that only a man has and that is located in the lower abdomen. This is a descriptive study, of the experience report type, carried out in a basic health unit in the State of Pará. men's resistance to continuing periodic consultations, therefore, an activity was carried out with the aim of developing the health-disease process, to meet the specific target audience of men of prostate cancer screening age. The intention was to acquire a greater number of adherence of patients from the male health unit to screening. Thus, multidisciplinary care is important so that all teaching axes can be sought to be passed on to the community, so that educational actions together clarify forms of prevention and provide an early diagnosis.
\end{abstract}

Keywords: Cancer; Health education; Multi-professional.

\section{Resumen}

El cáncer de próstata es el tipo más común entre los hombres. La próstata es una glándula que solo tiene un hombre y que se ubica en la parte inferior del abdomen. Se trata de un estudio descriptivo, tipo relato de experiencia, realizado en una unidad básica de salud en el Estado de Pará. Resistencia de los hombres a las consultas periódicas continuas, por lo que se realizó una actividad con el objetivo de desarrollar el proceso salud-enfermedad, para cumplir con el público objetivo específico de hombres en edad de detección del cáncer de próstata. La intención era adquirir un mayor número de pacientes de la unidad de salud masculina al cribado. Por ello, la atención multidisciplinar es importante para que se puedan buscar todos los ejes didácticos para trasladarlos a la comunidad, de modo que las acciones educativas en conjunto clarifiquen formas de prevención y proporcionen un diagnóstico precoz.

Palabras clave: Cáncer; Educación para la salud; Multi-profesional.

\section{Introdução}

A próstata é uma glândula que faz parte do sistema reprodutivo masculino, se situa abaixo da bexiga, envolvendo a uretra e na frente do reto. A glândula prostática possui a função de eliminar a urina que se acumula na bexiga, e é também responsável pela produção do esperma que conduz os espermatozoides pelo canal uretral (Krüger \& Cavalcanti, 2018).

A doença neoplásica se apresenta como uma grave problemática de saúde pública a nível mundial, onde se constitui uma relação estreita com as complicações do metabolismo, imunologia e genética, sendo em estado basal ou em repouso (Nogueira \& Lima, 2018).

De acordo com o Instituto Nacional do Câncer (INCA, 2021) no Brasil, o câncer de próstata é o segundo mais comum entre os homens, atrás apenas do câncer de pele não - melanoma. Mais do que qualquer outro tipo, é considerado um câncer da terceira idade, já que cerca de $75 \%$ dos casos do mundo ocorrem a partir dos 65 anos. Alguns desses tumores podem crescer de forma rápida, espalhando-se para outros órgãos e podendo levar à morte

A Política Nacional de Atenção Integral à Saúde do Homem, lançada pelo Ministério da Saúde, veio com o objetivo de facilitar e ampliar o acesso da população masculina aos serviços de saúde, em resposta à observação de que os agravos do sexo masculino são um problema de saúde pública (Oliveira et al.,2019). 
O câncer de próstata pode apresentar evolução silenciosa no início, os pacientes podem não apresentar sintomas ou, podem apresentar sintomas parecidos aos do tumor benigno da próstata. No estágio mais avançado pode causar alguns sintomas, como: micção frequente, fluxo urinário fraco ou interrompido, sangue na urina ou no sêmen, dor no quadril, costas, coxas, ombros ou outros ossos se a doença se disseminou, e fraqueza ou dormência nas pernas (Junior et al.,2015).

O exame toque retal é uma medida de prevenção que possui baixo custo, porém, em alguns casos, muda a ideação masculina afastando essa população da realização do mesmo, não apenas pela escassez de informação, mas também pela simbologia e pela vergonha, bem como questões psicológicas e emocionais ou estruturas de acesso a esse tipo de exame pela rede pública de saúde (Moraes \& Silva, 2017).

Damião et al. (2017) afirma que, o toque retal apesar de desconfortável e constrangedor, ainda constitui uma importante ferramenta no diagnóstico e estadiamento do câncer de próstata, já que cerca de 80\% dos tumores encontram-se na zona periférica da glândula prostática. O toque retal e o PSA (Antígeno Prostático Específico) auxiliam para rastrear o câncer de próstata, as alterações nesses exames levantam suspeita e podem servir para realização de uma biópsia que é uma forma de diagnóstico do câncer.

O exame de toque prostático serve para avaliar seu tamanho, formato e consistência, podem ser constatadas alterações nas porções posterior e lateral da próstata. Esse exame associado com a dosagem do PSA, podem reforçar a suspeita do diagnóstico de neoplasia, pois é dosado a quantidade de antígeno produzido pelas células epiteliais da próstata (Moreira, 2012).

Fatores ambientais e alimentares, incluindo alta ingesta de carne vermelha, gorduras, leite e alto consumo energético são fatores considerados influenciadores para o desenvolvimento dessa neoplasia. Outros fatores de risco que podem estar relacionado ao desenvolvimento de tumores malignos na próstata, são idade, raça, história familiar de câncer, níveis hormonais, exposição a agentes químicos e elementos oncogênicos, tais como supressores de tumor, genes mutados e antígenos que aumentam a suscetibilidade dos mesmos (Toebe et al.,2020).

Segundo o INCA (2021), em sua fase inicial o câncer de próstata tem evolução silenciosa, muitos pacientes não apresentam nenhum sintoma, ou quando apresentam são semelhantes aos do crescimento benigno da próstata, como dificuldade de urinar, necessidade de urina mais veze durante o dia ou á noite. Na fase avançada, pode provocar dor óssea, sintomas urinários ou, quando mais grave, infecção generalizada ou insuficiência renal.

De acordo com Quijada et al. (2017) o câncer de próstata é uma preocupação para a saúde pública, principalmente em países desenvolvidos, pois aumenta em concomitância com a expectativa de vida da população masculina. Neste ponto de vista o câncer de próstata quando diagnosticado precocemente, possui altas chances de cura, desde que se inicie um tratamento de imediato (Biondo et al., 2020).

O intuito deste trabalho, é relatar o desempenho acadêmico, para que desde já possamos ter habilidades para trabalhar como uma equipe multiprofissional, a fim de proporcionar saúde e bem estar para o paciente. Para obter sucesso na prevenção e tratamento a população deve ser melhor esclarecida sobre a importância da realização dos exames preventivos.

\section{Metodologia}

Este estudo caracteriza-se como descritivo, do tipo relato de experiência, realizado em uma Unidade Básica de Saúde, no Estado do Pará, no período de novembro de 2021.

Durante as atividades multiprofissionais notou-se a importância e a falta de informação relacionada ao câncer de próstata. Na UBS, sucedeu a conscientização do tema mencionado, que se deu juntamente aos profissionais da referida unidade que já atuam na área, com intuito de repassar aos pacientes que aguardavam pela consulta um pouco sobre o câncer de próstata, prevenção e suas formas de tratamento. 
A metodologia empregada transcorreu pela teoria da problematização, através do Arco de Maguerez no qual se trata de uma ferramenta que trabalha na perspectiva do ensino pela problematização, composta por cinco etapas que visam o estudo de uma determinada realizada para posterior intervenção, dividindo-se em: observação da realidade, levantamento de pontos chave, teorização, desenvolvimento das hipóteses de solução, e aplicação à realidade (Berbel, 2011).

Primeiramente notou-se que os homens têm uma baixa adesão ao exame, além disso durante as consultas observou a desinformação dos homens quando indagados sobre a realização do exame, bem como a pouca compreensão sobre a sua importância.

No entanto, optou-se em trabalhar junto aos profissionais que já atuam na UBS, este assunto sobre câncer de próstata dentro da unidade, visto que o local é propício para o desenvolvimento de ações educativas.

Foi trabalhado tecnologias leves, por meio da utilização de cartazes e dinâmica interativa, para que os homens que aguardavam consultas participassem e uma forma clara e transparente, para que todos pudessem participar da dinâmica, a fim de repassar conhecimento acerca do assunto, e os mesmo sanarem suas dúvidas, caso necessário.

A dinâmica ocorreu em todas as partes do posto de saúde, onde havia homens aguardando pelas consultas que estavam tendo no dia. A utilização de um elemento que chame atenção do público, se torna primordial para que conseguíssemos alcançar a atenção e conhecimentos dos mesmo para o assunto abordado.

A base teórica transcorreu pela busca bibliográfica em bases de dados científicas digitais, sendo elas: Scientific Eletronic Library Online (SCIELO) e Biblioteca Virtual em Saúde (BVS), com a utilização de palavras chaves apanhadas nos Descritores em Ciências da Saúde (DeCS), sendo elas:

\section{Resultados e Discussão}

A saúde é uma ciência que tem como principal objetivo, o cuidado. Nessa perspectiva o enfoque preventivo, assim como a promoção da saúde, deve nortear a prática assistencial. (Soares \& Heidemann, 2019).

A promoção em saúde é considerada um campo conceitual e de práxis, e tem influenciado a organização do sistema de saúde de diversos países e regiões do mundo. A promoção em saúde é a intervenção sobre as condições de vida da população (Mascarenha et al.,2012).

Sendo assim, a participação da equipe multiprofissional é essencial na abordagem, visto que cada um com seus conhecimentos científicos contribuem para a promoção de saúde.A importância de discutir este assunto na atenção primária é importante para uma detecção precoce, visto que alguns homens podem sentir desconforto e não procuram atendimento médico por falta de informação e até mesmo pelo preconceito em relação aos exames prescritos para detecção precoce.

A equipe multiprofissional tanto para detecção precoce como para o tratamento do câncer de próstata é fundamental na vida do paciente. Segundo o INCA (2021) o objetivo da atenção primária é evitar que o câncer se desenvolva, a fim de preservar a exposição de fatores de risco e adoção de uma vida saudável.

No decorrer da pesquisa teórica e prática, podemos notar a falta de esclarecimento sobre o câncer de próstata na atenção primária em relação à prevenção, diagnóstico e tratamento. Sendo assim, a proposta realizada tem a intenção de informar esses homens sobre o câncer de próstata e deter os estigmas impostos pela sociedade.

O medo da doença, dor e morte é considerado como uma das principais motivações para que os homens busquem a realização de exames preventivos e diagnósticos para avaliar seu estado de saúde (Morais et al.,2020).

Durante a exposição do cartaz e da prática dinâmica, foi possível repassar para esses homens, sobre o que é o câncer de próstata, suas formas de prevenção e tratamento. Possibilitando evitar estigmas que ainda cercam a sociedade masculina, sobre os exames que precisam ser realizados. 
Resumidamente, a dinâmica sucedeu da seguinte maneira: foi entregue placas únicas com lados anterior e posterior nas cores verde que corresponde a " verdadeiro", e cores vermelhas que correspondia a "falso".

Após toda a explicação sobre o que era câncer de próstata, sinais e sintomas, diagnóstico e tratamento, foram realizadas perguntas que abordavam esses eixos citados, e os mesmo com as placas, deveriam responder se aquela pergunta era verdadeira ou falsa, a fim de observar a interação dos mesmos, e se eles tinham absorvido a importância de principalmente realizar o exame.

A educação em saúde é uma estratégia que busca disponibilizar aos indivíduos conhecimentos e habilidades que auxiliem nas escolhas sobre sua saúde, instigando a consciência crítica, reconhecendo assim os fatores que influenciam a saúde e oferecendo subsídios que os encorajem a modificar o comportamento, baseado na interação respeitosa da cultura popular com os saberes científicos (Júnior et al., 2020).

Outrossim, foi elucidado a questão do diagnóstico e consultas periódicas, visto que os homens têm uma certa resistência em seguir esses exames e consultas de rotina. Levando em consideração a importância de um atendimento multiprofissional, envolvendo médicos, enfermeiro, nutricionista, entre outros

Além dos esclarecimentos e informações diante do tema, durante a roda de conversa notou-se que o público alvo tinha um conhecimento básico sobre o câncer de próstata, mas era fundamental alertá-los sobre os sinais e sintomas, prevenção e tratamento.

Destaca-se o desempenho acadêmico, que permite contato mais próximo com o paciente por base a meio de práticas didáticas, retratando temas como este, de forma recreativa, tendo como finalidade repassar os conhecimentos obtidos de maneira clara.

De acordo com o desenvolvimento das ações educativas, podemos analisar a importância da atenção em saúde ao público alvo, que permitiu-nos observar o engajamento e despertar a curiosidade. Visto que exista uma baixa adesão na procura quando relaciona-se ao preconceito e a masculinidade possam mostrar-se com maior evidência ainda nos dias atuais.

Como demonstração do conhecimento, após a realização da atividade de conscientização, foi percebido através de análise no momento pós - atividade. A integração da educação em saúde na unidade de saúde da família, teve como capacidade a conscientização. Por conseguinte, foi capaz de realizar o objetivo que era conscientizar e explicar a esses homens em uma linguagem acessível e de simples entendimento, para que possamos diminuir os casos de incidência causados pelo câncer de próstata, além de conseguir mais uma adesão dos mesmos, para realizar o exame que apesar de ainda ser um estigma pela sociedade, é considerado a melhor forma de prevenção.

\section{Considerações Finais}

Em síntese, é importante a abordagem deste tema na atenção primária, visto que é porta de entrada para muitas pessoas para detecção de doenças. As ações educativas relacionadas à conscientização se tornam necessárias, a fim de estimular esses homens a procurarem tratamento ao notarem qualquer sintoma.

Ademais, constatou-se que atividades dinâmicas proporcionam a escuta e fala dos usuários, promovendo sua autonomia.

Sendo assim, o estudo teve como objetivo relatar uma experiência sobre educação em saúde, pois acredita-se que a educação em saúde remete reflexões e intervenções de expansão de conhecimento

Vale salientar, a junção das diversas áreas da saúde, promovendo ações voltadas para a comunidade, sendo indispensáveis para criação de novas estratégias para melhorar a adesão.

Por fim, evidenciou-se a partir dos resultados obtidos que o objetivo foi alcançado, visto que houve interação e reflexão a respeito do tema, o que permitiu que os pacientes fossem capazes de opinar nas decisões de saúde para cuidar de si e da coletividade. 
Vale ressaltar também, que sejam incluídas novas atividades de educação em saúde, no contexto de atenção à saúde primária, e novos estudos nessa área de concentração, pois a relevância de estudos como estes são importantes para a sociedade.

\section{Referências}

Berbel, N. A. N. (2011). As metodologias ativas e a promoção da autonomia de estudantes. Semina: Ciências Sociais e Humanas, 32(1), 25-40.

Biondo, C. S., Santos, J. d., Ribeiro, B. S., Passos, R. d. S., Meira, A. P. B. N., \& Soares, C. d. J. (2020). Detecção precoce do câncer de prostáta: atuação da equipe de saúde da familia., 1-13.

Câncer, I. N. d. (2021). Câncer de próstata. 1-5

Damião, R., Figueiredo, R., Dornas, M., Lima, D., \& Koschorke, M. (2017). Câncer de próstata. 1-7.

Júnior, A. J. B., Menezes, C. S., Barbosa, C. D. A., Freitas, G. B. S., Silva, G. G., Vaz, J. P. S., Souza, M. L., \& Oliveira, T. M. (2015). Câncer de próstata: métodos de diagnóstico, prevenção e tratamento. 1-7.

Júnior, A. M. d. F., Cunha, F. F. d., Azevedo, B. A. R. d., \& Castro, H. S. d. (2020). Percepção de acadêmicos de Enfermagem sobre educação em saúde na perspectiva da qualificação do cuidado. 1-7.

Krüger, F. P. G., \& Cavalcanti, G. (2018). Conhecimento e Atitudes sobre o Câncer de Próstata no Brasil: Revisão Integrativa. 1-7

Mascarenhas, N. B., Melo, C. M. M. d., \& Fagundes, N. C. (2012). Produção do conhecimento sobre promoção da saúde e prática da enfermeira na Atenção Primária., 1-9.

Moraes, M. C., da Costa Oliveira, R., \& Silva, M. D. J. (2017). Uma questão masculina: conhecendo possíveis entraves para a realização dos exames de detecção do câncer de próstata. Revista Medica Herediana,28(4), 230-235.

Moreira, N. M. (2012). O preconceito em relação ao exame de toque retal como forma de rastreamento do câncer de prostáta., 3-25.

Morais, R. L. G. L., Tosta, M. S., Santos, J., \& Oliveira, J. d. S. (2020). Conhecimento dos homens sobre o câncer de próstata: a virilidade e o estigma da doença. 1-7

Nogueira, H. S., \& Lima, W. P. (2018). Câncer, sistema imunológico e exercício físico: uma Revisão narrativa. Corpoconsciência,22(1), 40-52.

Oliveira, P. S. D., Miranda, S. V. C. d., Barbosa, H. A., Rocha, R. M. B. d., Rodrigues, A. B., \& Silva, V. M. d. (2019). Câncer de próstata: conhecimentos e interferências na promoção e prevenção da doença. 1-12

Quijada, P. D. d. S., Fernandes, P. A., Oliveira, D. S. d., \& Santos, B. M. d. O. (2017). Câncer de próstata: retrato de uma realidade de pacientes em tratamento. 1-10.

Soares, C. F., \& Heidemann, I. T. S. B. (2019). Promoção Da Saúde E Prevenção Da Lesão Por Pressão: Expectativas Do Enfermeiro Da Atenção Primária. $1-9$.

Toebe, A. L., Figueró, N. A., Buhring, C. A. Z., \& Mendes, G. A. (2020). Câncer de próstata: revisão da literatura acerca dos diversos aspectos da doença. $1-4$. 